\title{
CORRESPONDENCE
}

\section{Is airway regional resistances array a neglected component of ventilation?}

\author{
J-François Hicter ${ }^{*}$ (D, Julien Drevon and Sylvain Vallier
}

(0) 2021 Springer-Verlag GmbH Germany, part of Springer Nature

First, we would like to thank Marini et al. for their innovative work about ventilation induced lung injuries (VILI), giving a prominent attention to flow rate and flow profile [1]. The demonstration of flow related power and energetical impact opens up new perspectives for the management of mechanical ventilation. The proposed modelling contrasted two typical lungs, the first one with acute respiratory distress syndrome (ARDS) known for high elastance, and the second one with chronic obstructive pulmonary disease (COPD), known for high resistance. A focus is made on the energetical aspects of insufflation and strain, while emphasising intracycle variability of mechanical effects. The results suggest that flow related powers are different, according to the lung considered, but also to the flow profile applied. In their conclusion, the authors propose, according to protective ventilation strategy, to reduce flow rate by reducing minute ventilation (VM), and optimizing I/E ratio. Unfortunately, in vivo modelling is confronted with complexity of fluid dynamic, especially for compressible fluid flow, turbulent conditions, and bronchial network with uncertain physical properties [2]. In some pathological conditions with heterogenous lungs, mechanical properties of both models described by the authors can coexist at the same time [3]. We think that lung damage can be divided at least into four theoretical areas, according to their elastance and resistance: lowR lowE, lowR highE, highR lowE, highR highE. The healthy part of the lung should be identified with low elastance and low resistance (lowE, lowR). To simplify, R relates to regional lower bronchial tree status, E relates to regional alveolar status. The resistive component effect is easy to highlight, using simple experimental conditions, by connecting together on a

*Correspondence: dr.HICTER@clinique-belledonne.fr

Département d'Anesthésie Réanimation, Clinique Belledonne, 83 Av G PERI, 38400 Saint-Martin-d'Hères, France ventilator two test lungs assuming same compliance, one with a large and short tube, and the second with straight and long tube: the more the flow rate is high, the less the distribution of a tidal volume between the two lungs is even, due to the flow-resistive component that increases airway energy dissipation in the straight tube. Regional distribution of energy, elastic power, strain and stress are uneven as well. On the graphs proposed by Marini, the difference between COPD (HighR lowE) and ARDS (HighE) is particularly strong for decelerating flow " Pset». In his analysis, the author also emphasises the role of the high flow rate. At the patient bedside, spirometry, and static measures by occlusion manoeuvres, do not allow to explore either intracycle alveolar phenomenon, or energy transmission. As described for the COPD model, power impact of high flow rate could be limited, in some cases, by regional conditions that enhance flow-energy dissipation, and limit alveolar elastic power transmission. So, it's possible that RR, flow rate, and minute volume limitation, do not benefit all ARDS patients, with regard to negative effects of severe hypercapnia or ECCO2R, if necessary. In our unit, we try to investigate the impact of flow-rate settings on lung aeration by using electrical impedance tomography (EIT). EIT allows to study regional aeration distribution with appropriate temporal resolution to explore intracycle dynamic aspects of lung ventilation (Regional Ventilation DelayRVD) [4]. The results suggest, for some patients, that aeration kinetic and regional distribution differ, depending on the flow rate setting. Today, we have few data available to predict intracycle power-induced damage. But despite technical limitations, EIT will probably succeed in helping clinicians in the management of ventilation settings, especially flow rate and flow profile, respiratory rate (RR), Ti, Te to provide the most homogenous aeration in terms of kinetic and regional distribution. Thus, obtaining impedance temporal uniformity (i.e., without RVD)

\section{Springer}


should be one good guaranty against alveolar aggression induced by regional flow and "elastic power" spikes.

\section{Declarations}

\section{Conflicts of interest}

The authors affirm that none has a conflict of financial or other interest relevant to this work.

\section{Publisher's Note}

Springer Nature remains neutral with regard to jurisdictional claims in published maps and institutional affiliations.

Received: 17 April 2021 Accepted: 3 May 2021

Published online: 18 May 2021

\section{References}

1. Marini JJ, Crooke PS, Gattinoni L (2021) Intra-cycle power: is the flow profile a neglected component of lung protection? Intensive Care Med. https://doi.org/10.1007/s00134-021-06375-5

2. Faizal WM, Ghazali NNN, Khor CY, Badruddin IA, Zainon MZ, Yazid AA, Ibrahim NB, Razi RM (2020) Computational fluid dynamics modelling of human upper airway: a review. Comput Methods Programs Biomed 196:105627

3. Chen IC, Kuo J, Ko W-J, Shih H-C, Kuo CD (2016) Increased flow resistance and decreased flow rate in patients with acute respiratory distress syndrome: the role of autonomic nervous modulation. J Chin Med Assoc 79:17-24

4. Shono A, Kotani T (2019) Clinical implication of monitoring regional ventilation using electrical impedance tomography. J Intensive Care 7:4 